\title{
Hydrogen Bond Networks in Binary Mixtures of Water and Organic Solvent SI
}

Simon Stehle*, and Andreas Siegfried Braeuer,**

*Institute of Thermal-, Environmental- and Resources‘ Process Engineering (ITUN),

Technische Universität Bergakademie Freiberg (TUBAF), Leipziger Strasse 28, 09599

Freiberg, Germany

$\S$ Erlangen Graduate School in Advanced Optical Technologies (SAOT), Friedrich-

Alexander-Universitaet Erlangen-Nuernberg (FAU), Paul-Gordan-Straße 6, 91052 Erlangen,

Germany

*andreas.braeuer@tu-freiberg.de

\section{Spectra processing}

\subsection{Baseline correction}

The raw experimental spectra are baseline corrected by subtracting an underground spectrum from the raw experimental spectrum. The underground spectrum is generated by fitting a spline function through points in the spectrum at which the Raman signal intensity, due to the absence of Raman peaks at these positions, is known to be zero. For further details we refer to reference. ${ }^{1}$

\subsection{Isolation of the $\mathrm{OH}$ stretching vibration}

In the baseline corrected Raman spectra $S_{m i x}^{e x p}$ of binary mixtures of water and organic solvent the $\mathrm{CH}$ stretching vibration of the organic solvent spectrally overlaps between $3000 \mathrm{~cm}^{-1}$ and $3200 \mathrm{~cm}^{-1}$ with the $\mathrm{OH}$ stretching vibration of water or of the alcohol. Therefore, we subtract according to equations (1) and (2) the $\mathrm{CH}$ signal from the mixture spectra. On this purpose the 
mixture spectrum $S_{\text {mix }}\left(\tilde{v}_{S}\right)$ is represented by a modelled mixture spectrum $S_{\text {mix,mod }}\left(\tilde{v}_{S}\right)$ that, using a partial least squares minimization, is fitted to the experimental one $S_{m i x}\left(\tilde{v}_{S}\right)$. The modelled spectrum

$$
S_{\text {mix,mod }}\left(\tilde{v}_{S}\right)=S_{\text {mod }}^{C H, 1}-4\left(\tilde{v}_{S}\right)+S_{\text {mod }}^{\text {OH, }}{ }^{6}\left(\tilde{v}_{S}\right),
$$

is composed of 4 pseudo Voigt profile peaks $S_{\text {mod }}^{C H, 1}-4$ that represent the $\mathrm{CH}$ Raman signal (dotted orange in Figure S1) and of six Gaussian profile peaks $S_{\text {mod }}^{O H, 1-6}$ (dotted blue in Figure S1) that represent the $\mathrm{OH}$ stretching vibration of the alcohol and water. In order to obtain the isolated $\mathrm{OH}$ Raman spectrum of the mixture

$$
S_{m i x}^{O H}\left(\tilde{v}_{S}\right)=S_{m i x}\left(\tilde{v}_{S}\right)-S_{m o d}^{C H, 1-4}\left(\tilde{v}_{S}\right)
$$

we subtract the modelled $S_{m o d}^{C H, 1}{ }^{-4} \mathrm{CH}$ Raman signal from the mixture spectrum $S_{\text {mix }}$.

During the fitting procedure of the modelled spectrum to the experimental spectrum each of the four pseudo Voigt profile peaks for the $\mathrm{CH}$ Raman signal and the six Gaussian profile peaks for the $\mathrm{OH}$ Raman signal have certain degrees of freedom. Their central peak position can be fitted within certain ranges (Table S1), whereas the peak height and their width have no restraints.

Table S1: List of restraints for the peak centers for the fit of the four pseudo Voigt profile peaks for the $\mathrm{CH}$ Raman signal and the six Gaussian profile peaks for the $\mathrm{OH}$ Raman signal. For acetonitrile $S_{\text {mod }}^{\mathrm{CH}, 1-4}$

\begin{tabular}{|c|c|c|}
\hline Peak & $\begin{array}{c}\text { Peak center } / \mathrm{cm}^{-1} \\
\text { Methanol }\end{array}$ & $\begin{array}{c}\text { Peak center } / \mathrm{cm}^{-1} \\
\text { Ethanol }\end{array}$ \\
\hline $\mathrm{CH} 1$ & $2550-2650$ & $2710-2730$ \\
\hline $\mathrm{CH} 2$ & $2835-2845$ & $2870-2890$ \\
\hline $\mathrm{CH} 3$ & $2942-2952$ & $2920-2940$ \\
\hline $\mathrm{CH} 4$ & $2950-3050$ & $2960-2980$ \\
\hline OH1 & \multicolumn{2}{|c|}{$3165-3250$} \\
\hline $\mathrm{OH} 2$ & \multicolumn{2}{|c|}{$3250-3300$} \\
\hline ОН3 & \multicolumn{2}{|c|}{$3320-3450$} \\
\hline $\mathrm{OH} 4$ & \multicolumn{2}{|c|}{$3450-3560$} \\
\hline OH5 & \multicolumn{2}{|c|}{$3560-3700$} \\
\hline OH6 & \multicolumn{2}{|c|}{$3700-3500$} \\
\hline
\end{tabular}
has been replaced by the pure $\mathrm{Acn} \mathrm{CH}$ spectrum peak, without fitting a model spectrum.

Additionally the shape of the pseudo Voigt profile peaks can be fitted by varying the fractions of Gaussian and Lorentzian shares. Due to the many fit parameters the partial least squares 
optimization algorithm requires quite some computational effort. The computational effort can be reduced, if the modelled CH Raman signal $S_{m o d}^{C H, 1-4}$ is once fitted to the pure compound spectrum of the organic solvent, as it is shown in Figure S1, and then considered as fixed peak assemble, where all fit parameters except the intensity of the peak assemble are kept constant.

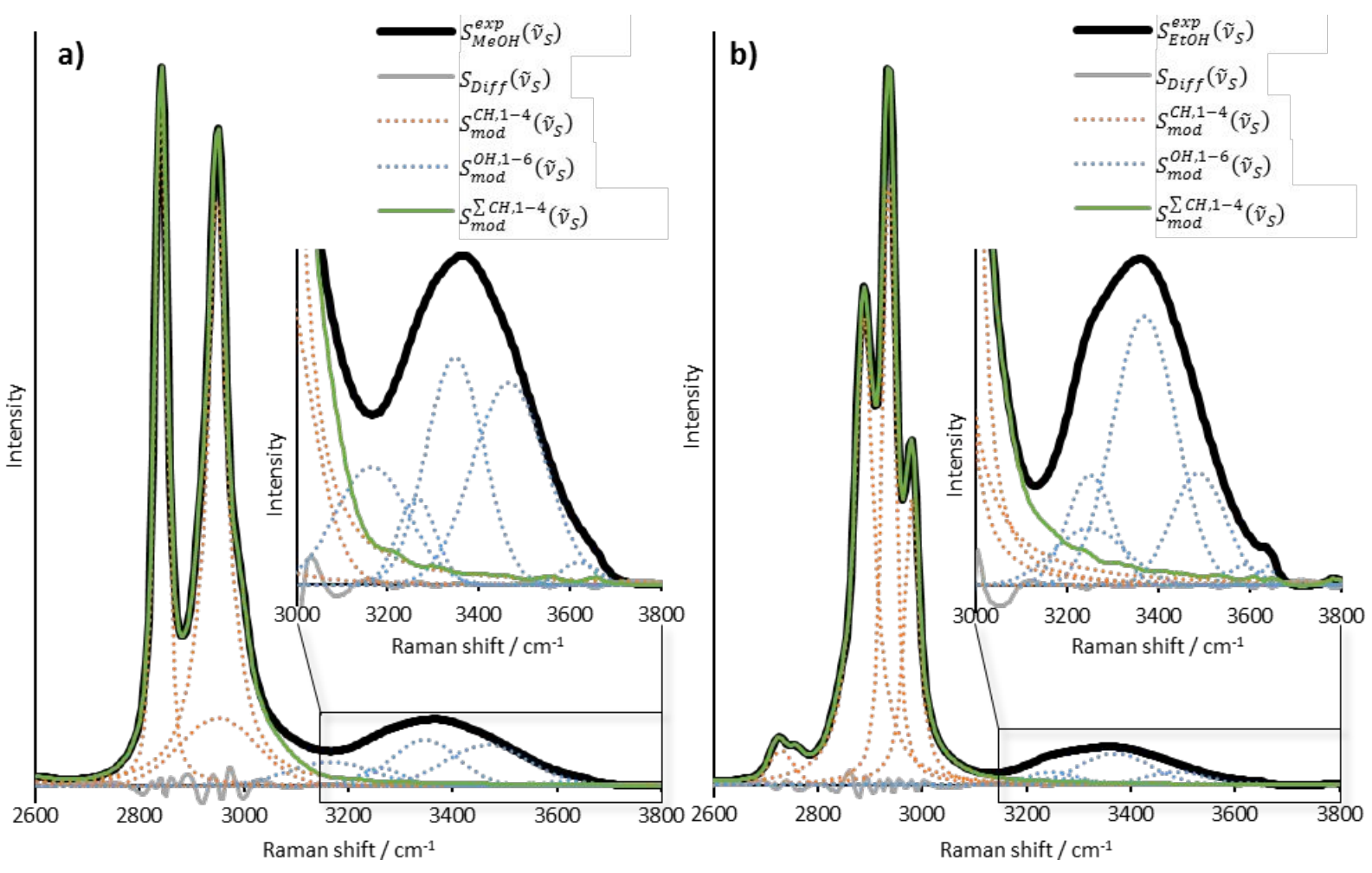

Figure S1: Raman spectra of pure methanol (a) and pure ethanol (b) at $0.4 \mathrm{MPa}$ and $308 \mathrm{~K}$ (bold black line) with the partial least square fit of 4 pseudo Voigt profiles for the $\mathrm{CH}$ stretching vibration (dotted orange lines) and 6 Gaussian profiles for the $\mathrm{OH}$ stretching vibration (dotted blue lines). The green line shows the sum of the four pseudo Voigt profile peaks. Grey line: difference between the best fit and the pure compound spectra.

Figure S2 shows the isolated $\mathrm{OH}$ Raman spectra $S_{m i x}^{O H}\left(\tilde{v}_{S}\right)$ of the three analyzed mixtures of water and organic solvent for mixture compositions between pure water and pure organic solvent. The up-and-down course of the spectra $S_{m i x}^{O H}\left(\tilde{v}_{S}\right)$ at the left margin of the Raman-shift scale is an artifact of the subtraction of the CH-Raman signal according to equation (2) above. This range of Raman shifts is not considered for the computation of the centroid of the $\mathrm{OH}$ Raman signal. 


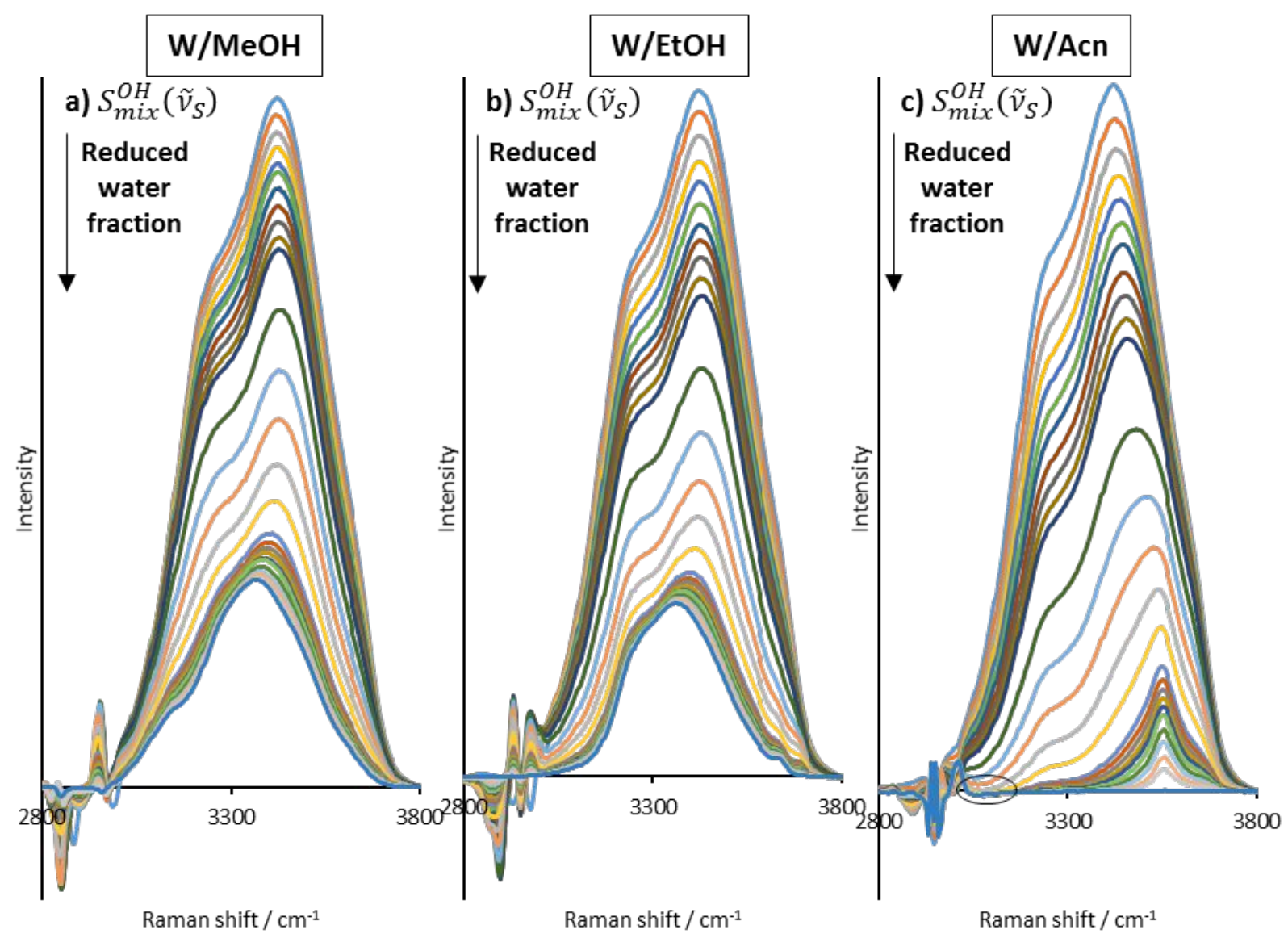

Figure S2: Isolated $\mathrm{OH}$ Raman spectra of the mixture $S_{\text {mix }}^{O H}\left(\tilde{v}_{S}\right)$ for the mixtures a) water/methanol, b) water/ethanol and c) water/acetonitrile at $0.4 \mathrm{MPa}$ and $308 \mathrm{~K}$ over the whole composition range. Pure water spectra show the larges intensity. The small black oval in (c) marks minimal negative intensity values resulting from the acetonitrile subtraction. We will discuss this at the calculation of the molar spectra were this effect becomes more visible. The mixture compositions for the all Raman spectra are listed in Table S2.

\subsection{Computation of molar Raman spectra}

The Raman spectra $S_{m i x}^{O H}\left(\tilde{v}_{S}\right)$ are converted into molar Raman spectra $s_{m i x}^{O H}\left(\tilde{v}_{S}\right)$

$$
s_{m i x}^{O H}\left(\tilde{v}_{S}\right)=S_{m i x}^{O H}\left(\tilde{v}_{S}\right) v_{m}^{m i x} .
$$

by multiplication with the molar volume of the mixture $v_{m}^{\text {mix }}$. The molar volume of each mixture

$$
v_{m}^{\text {mix }}=\frac{M_{\text {mix }}}{\rho_{\text {mix }}}=\frac{x_{1} M_{1}^{0}+x_{2} M_{2}^{0}}{\rho_{\text {mix }}}
$$

is computed from the density of the mixture $\rho_{\text {mix }}$ which is determined with the Coriolis densitometer, the respective molar fractions $x_{i}$ of the compounds in the mixture, which are known from the amounts of the compounds fed via the syringe pumps, and their molar masses $M$. The molar Raman spectra $S_{m i x}^{O H}\left(\tilde{v}_{S}\right)$ of mixtures water/organic solvent are presented in Figure S3. The centroid is computed from portion of $S_{m i x}^{O H}$ inside the blue background rectangle. 


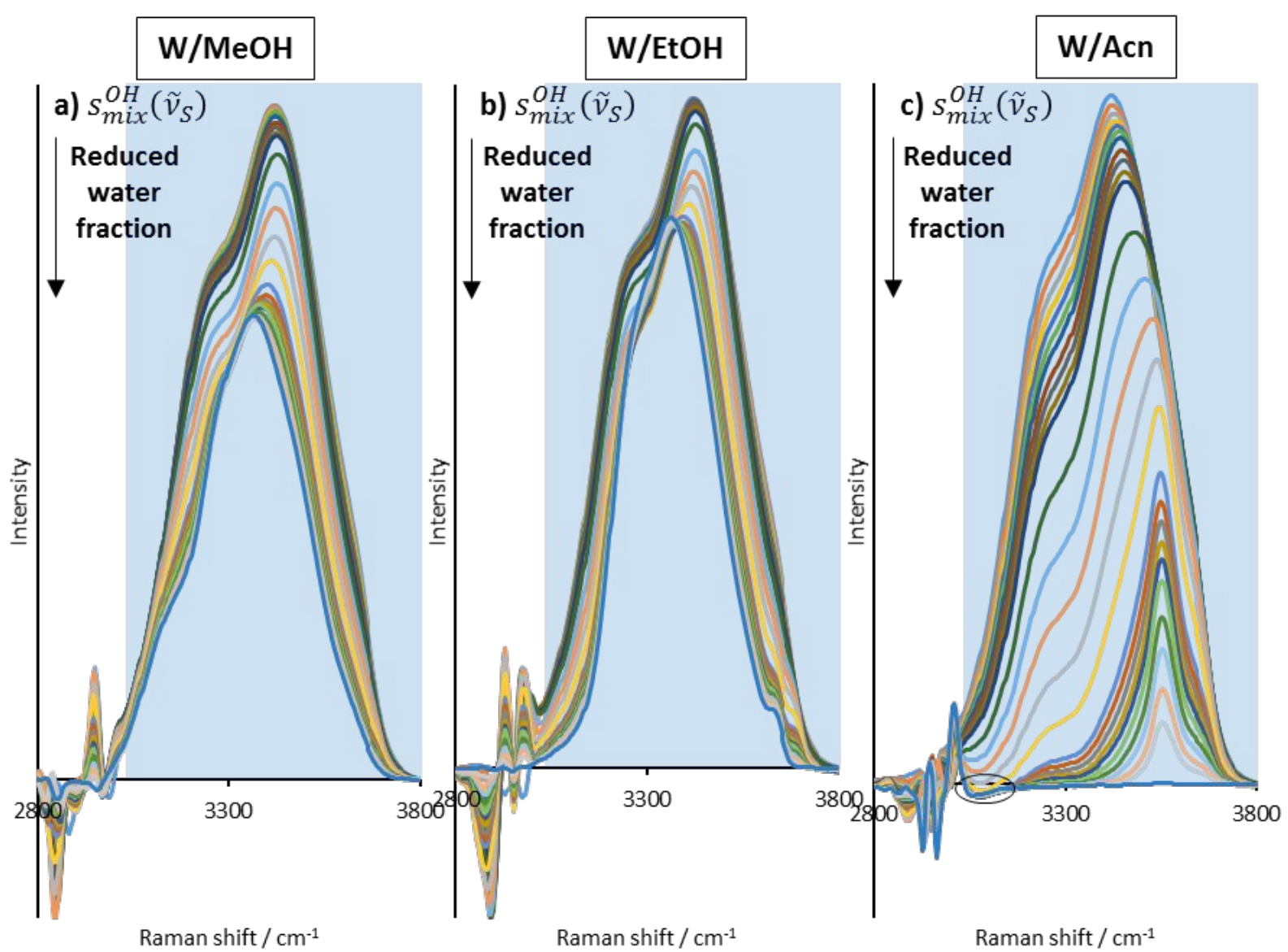

Figure S3: Molar OH Raman spectra $S_{m i x}^{O H}\left(\tilde{v}_{S}\right)$ of the mixtures a) water/methanol, b) water/ethanol and c) water/acetonitrile at $0.4 \mathrm{MPa}$ and $308 \mathrm{~K}$ over the whole composition range. The blue background illustrates the area from which the centroid is calculated. The small black oval in (c) marks minimal negative intensity values resulting from the acetonitrile subtraction. The mixture compositions for the all Raman spectra are listed in Table S2.

\subsection{Computation of partial molar spectra}

The partial molar Raman spectra of compound $i$ in the mixture with compound $\mathrm{j}$

$$
\bar{s}_{i(j)}^{O H}\left(\tilde{v}_{S}\right)=s_{m i x}^{O H}\left(\tilde{v}_{S}\right)+\left(1-x_{i}\right)\left(\frac{\partial s_{m i x}^{O H}\left(\tilde{v}_{S}\right)}{\partial x_{i}}\right)_{T, p}
$$

and of compound $\mathrm{j}$ in the mixture with compound $i$

$$
\bar{s}_{j(i)}^{O H}\left(\tilde{v}_{S}\right)=s_{m i x}^{O H}\left(\tilde{v}_{S}\right)-x_{i}\left(\frac{\partial s_{m i x}^{O H}\left(\tilde{v}_{S}\right)}{\partial x_{i}}\right)_{T, p}
$$

are computed from the molar mixture spectra $s_{m i x}^{O H}\left(\tilde{v}_{S}\right)$ in accordance to the computation of thermodynamic partial molar properties ${ }^{2}$ In order to achieve an accurate description of the derivatives $\left(\frac{\partial s_{m i x}^{O H}\left(\tilde{v}_{S}\right)}{\partial x_{i}}\right)_{T, p}$ high quality Raman spectra have to be recorded in rather small increments with respect to the composition $x_{i}$. 
In practice, we apply equation (5) and (6) pixel by pixel or Raman shift by Raman shift. For example for a Raman shift of $3300 \mathrm{~cm}^{-1}$ the evolution of signal intensity $s_{m i x}^{O H}(3300)$ is considered as a function of the mixture composition and thereafter derived. Once this has been done for each pixel or for each Raman shift, the partial molar Raman spectrum can be reassembled for all Raman shifts.. Figure S4 shows in the upper row partial molar Raman spectra of water and in the bottom row the partial molar Raman spectra of the organic solvent.
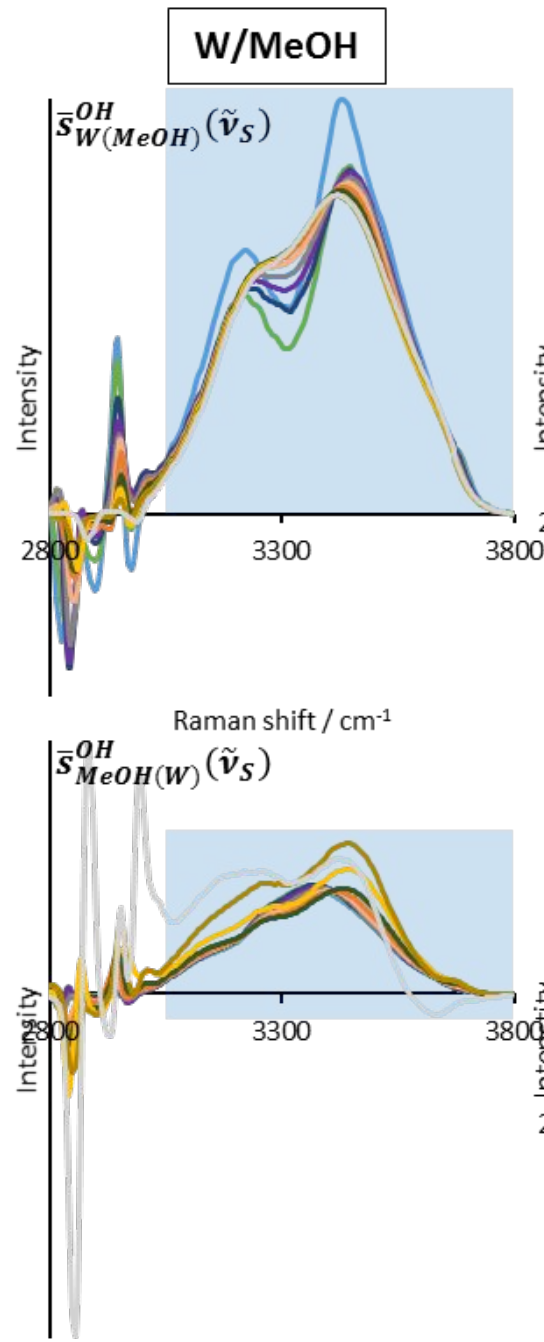

Raman shift $/ \mathrm{cm}^{-1}$

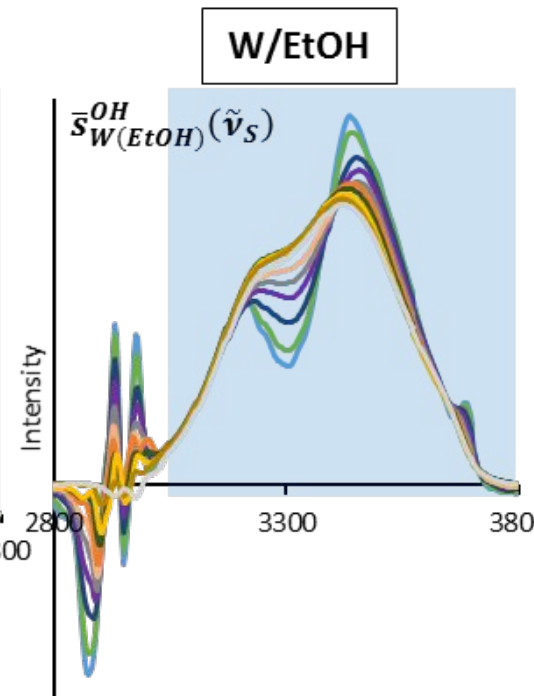

Raman shift $/ \mathrm{cm}^{-1}$

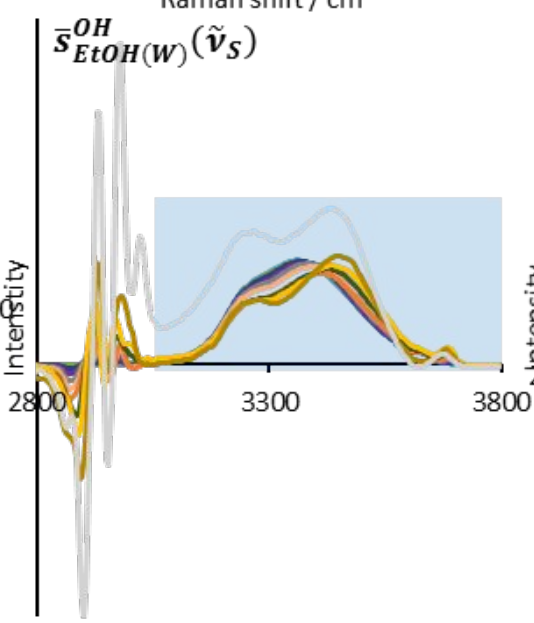

Raman shift $/ \mathrm{cm}^{-1}$

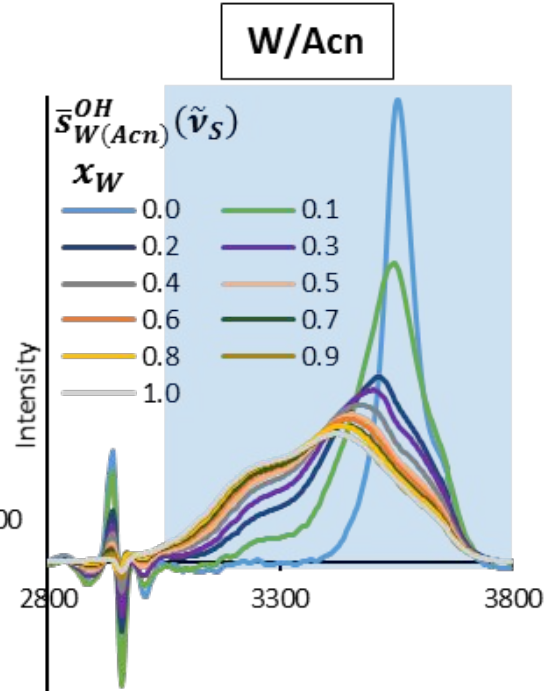

Raman shift $/ \mathrm{cm}^{-1}$

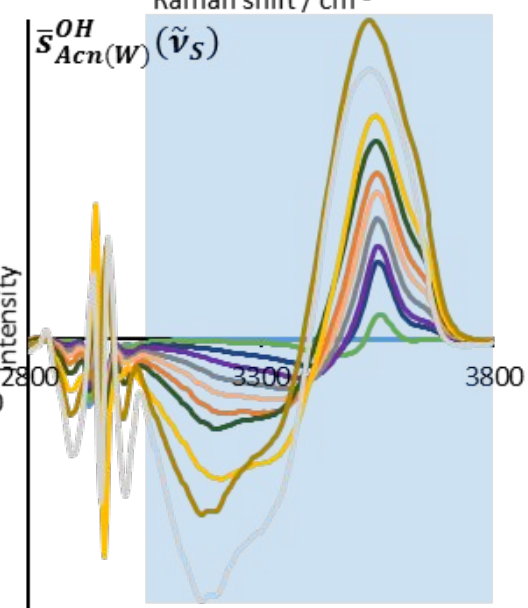

Raman shift $/ \mathrm{cm}^{-1}$

Figure S4: Partial molar $\mathrm{OH}$ Raman spectra of the three analyzed mixtures water/methanol, water/ethanol and water/acetonitrile (left to right) at $0.4 \mathrm{MPa}, 308 \mathrm{~K}$ and different water molar fractions. The upper row presents the partial molar spectra of water and the lower row the ones of the organic solvents. The blue background illustrates the area from which the centroid is calculated.

Negative intensity values within the partial molar Raman spectra are meaningful. They imply that for example the addition of acetonitrile to the mixture with water causes a reduction of the molar $\mathrm{OH}$ Raman spectrum $s_{m i x}^{O H}\left(\tilde{v}_{S}\right)$ at Raman shifts, where $\bar{s}_{A c n(W)}^{O H}\left(\tilde{v}_{S}\right)$ is negative and an increase where $\bar{s}_{A c n(W)}^{O H}\left(\tilde{v}_{S}\right)$ is positive. 


\section{$2 \quad$ Analyzed mixture compositions and densities}

Table S2: List of all investigated compositions and measured densities for the three binary mixtures W/Acn, W/MeOH and W/EtOH

\begin{tabular}{|c|c|c|c|c|c|c|}
\hline \multirow[b]{2}{*}{$\dot{V}_{A c n} / \mu \mathrm{l} \min ^{-}$} & \multirow[b]{2}{*}{$\begin{array}{c}\dot{V}_{W} / \\
\mu l \min ^{-1}\end{array}$} & \multicolumn{2}{|c|}{ water / acetonitrile } & \multirow[b]{2}{*}{$\begin{array}{c}\rho_{\operatorname{mix}}^{308 K} / \\
\mathrm{kg} \mathrm{m}^{-3} \\
\end{array}$} & \multirow[b]{2}{*}{$\begin{array}{c}\rho_{\operatorname{mix}}^{318 K} / \\
\mathrm{kg} \mathrm{m}^{-3}\end{array}$} & \multirow[b]{2}{*}{$\begin{array}{r}\rho_{\text {mix }}^{328 K} / \\
\mathrm{kg} \mathrm{m}^{-3} \\
\end{array}$} \\
\hline & & $x_{A c n}$ & $x_{W}$ & & & \\
\hline 0 & 200 & 0.00 & 1.00 & 999 & 998 & 997 \\
\hline 11 & 190 & 0.02 & 0.98 & 990 & 988 & 990 \\
\hline 22 & 180 & 0.04 & 0.96 & 985 & 980 & 980 \\
\hline 31 & 168 & 0.06 & 0.94 & 977 & 972 & 968 \\
\hline 41 & 162 & 0.08 & 0.92 & 968 & 963 & 959 \\
\hline 50 & 155 & 0.10 & 0.90 & 957 & 953 & 951 \\
\hline 58 & 147 & 0.12 & 0.88 & 951 & 946 & 940 \\
\hline 67 & 142 & 0.14 & 0.86 & 944 & 937 & 935 \\
\hline 72 & 130 & 0.16 & 0.84 & 936 & 929 & 927 \\
\hline 77 & 121 & 0.18 & 0.82 & 927 & 921 & 918 \\
\hline 84 & 116 & 0.20 & 0.80 & 919 & 914 & 908 \\
\hline 112 & 90 & 0.30 & 0.70 & 887 & 880 & 874 \\
\hline 128 & 66 & 0.40 & 0.60 & 861 & 853 & 847 \\
\hline 145 & 50 & 0.50 & 0.50 & 838 & 830 & 823 \\
\hline 161 & 37 & 0.60 & 0.40 & 819 & 811 & 804 \\
\hline 176 & 26 & 0.70 & 0.30 & 803 & 794 & 787 \\
\hline 186 & 16 & 0.80 & 0.20 & 789 & 780 & 773 \\
\hline 195 & 15 & 0.82 & 0.18 & 786 & 778 & 771 \\
\hline 200 & 13 & 0.84 & 0.16 & 784 & 775 & 768 \\
\hline 200 & 11 & 0.86 & 0.14 & 781 & 773 & 766 \\
\hline 170 & 8 & 0.88 & 0.12 & 779 & 771 & 764 \\
\hline 182 & 7 & 0.90 & 0.10 & 777 & 769 & 762 \\
\hline 210 & 6 & 0.92 & 0.08 & 774 & 766 & 758 \\
\hline 190 & 4 & 0.94 & 0.06 & 773 & 764 & 756 \\
\hline 230 & 3 & 0.96 & 0.04 & 771 & 762 & 754 \\
\hline 230 & 2 & 0.98 & 0.02 & 769 & 761 & 753 \\
\hline 200 & 0 & 1.00 & 0.00 & 768 & 759 & 752 \\
\hline
\end{tabular}

\begin{tabular}{|c|c|c|c|c|c|c|}
\hline \multicolumn{7}{|c|}{ water / methanol } \\
\hline $\begin{array}{l}\dot{V}_{M e O H} / \\
\mu l \min ^{-1}\end{array}$ & $\dot{V}_{W} / \mu \mathrm{l} \min ^{-1}$ & $x_{\text {МеOH }}$ & $x_{W}$ & $\begin{array}{c}\rho_{\operatorname{mix}}^{308 K} / \\
\mathrm{kg} \mathrm{m}^{-3}\end{array}$ & $\begin{array}{c}\rho_{\operatorname{mix}}^{318 K} / \\
\mathrm{kg} \mathrm{m}^{-3}\end{array}$ & $\begin{array}{c}\rho_{\text {mix }}^{328 K} / \\
\mathrm{kg} \mathrm{m}^{-3}\end{array}$ \\
\hline 0 & 200 & 0.00 & 1.00 & 999 & 998 & 997 \\
\hline 9 & 200 & 0.02 & 0.98 & 991 & 991 & 990 \\
\hline 18 & 190 & 0.04 & 0.96 & 985 & 985 & 984 \\
\hline
\end{tabular}




\begin{tabular}{ll|ll|lll}
25 & 175 & 0.06 & 0.94 & 979 & 979 & 977 \\
32 & 164 & 0.08 & 0.92 & 974 & 973 & 972 \\
40 & 160 & 0.10 & 0.90 & 971 & 970 & 965 \\
49 & 160 & 0.12 & 0.88 & 965 & 962 & 959 \\
55 & 150 & 0.14 & 0.86 & 959 & 958 & 955 \\
62 & 145 & 0.16 & 0.84 & 954 & 954 & 949 \\
69 & 140 & 0.18 & 0.82 & 949 & 948 & 944 \\
73 & 130 & 0.20 & 0.80 & 944 & 941 & 939 \\
101 & 105 & 0.30 & 0.70 & 921 & 918 & 913 \\
120 & 80 & 0.40 & 0.60 & 897 & 894 & 888 \\
139 & 62 & 0.50 & 0.50 & 875 & 870 & 866 \\
159 & 47 & 0.60 & 0.40 & 853 & 849 & 843 \\
168 & 32 & 0.70 & 0.30 & 833 & 828 & 823 \\
180 & 20 & 0.80 & 0.20 & 814 & 808 & 803 \\
185 & 18 & 0.82 & 0.18 & 810 & 805 & 799 \\
189 & 16 & 0.84 & 0.16 & 807 & 801 & 795 \\
193 & 14 & 0.86 & 0.14 & 803 & 797 & 791 \\
198 & 12 & 0.88 & 0.12 & 799 & 793 & 787 \\
202 & 10 & 0.90 & 0.10 & 796 & 789 & 784 \\
208 & 8 & 0.92 & 0.08 & 792 & 785 & 780 \\
210 & 6 & 0.94 & 0.06 & 789 & 782 & 776 \\
214 & 4 & 0.96 & 0.04 & 785 & 778 & 773 \\
216 & 2 & 0.98 & 0.02 & 782 & 775 & 769 \\
200 & 0 & 1.00 & 0.00 & 778 & 772 & 766
\end{tabular}

\begin{tabular}{|c|c|c|c|c|c|c|}
\hline \multicolumn{7}{|c|}{ water / ethanol } \\
\hline $\begin{array}{l}\dot{V}_{E t O H} / \\
\mu l \min ^{-1}\end{array}$ & $\dot{V}_{W} / \mu \mathrm{l} \min ^{-1}$ & $x_{E t O H}$ & $x_{W}$ & $\begin{array}{c}\rho_{\operatorname{mix}}^{308 K} / \\
\mathrm{kg} \mathrm{m}^{-3}\end{array}$ & $\begin{array}{c}\rho_{\operatorname{mix}}^{318 K} / \\
\mathrm{kg} \mathrm{m}^{-3}\end{array}$ & $\begin{array}{c}\rho_{m i x}^{328 K} / \\
\mathrm{kg} \mathrm{m}^{-3}\end{array}$ \\
\hline 0 & 200 & 0.00 & 1.00 & 999 & 998 & 997 \\
\hline 12 & 185 & 0.02 & 0.98 & 989 & 989 & 988 \\
\hline 24 & 180 & 0.04 & 0.96 & 980 & 980 & 980 \\
\hline 34 & 165 & 0.06 & 0.94 & 973 & 973 & 971 \\
\hline 45 & 160 & 0.08 & 0.92 & 966 & 964 & 962 \\
\hline 53 & 148 & 0.10 & 0.90 & 959 & 957 & 955 \\
\hline 61 & 138 & 0.12 & 0.88 & 953 & 951 & 948 \\
\hline 67 & 127 & 0.14 & 0.86 & 946 & 943 & 941 \\
\hline 75 & 122 & 0.16 & 0.84 & 939 & 937 & 932 \\
\hline 82 & 115 & 0.18 & 0.82 & 933 & 930 & 925 \\
\hline 90 & 111 & 0.20 & 0.80 & 926 & 923 & 918 \\
\hline 115 & 83 & 0.30 & 0.70 & 898 & 893 & 888 \\
\hline 138 & 64 & 0.40 & 0.60 & 872 & 867 & 861 \\
\hline 152 & 47 & 0.50 & 0.50 & 851 & 845 & 840 \\
\hline
\end{tabular}




\begin{tabular}{ll|ll|lll}
165 & 34 & 0.60 & 0.40 & 833 & 826 & 821 \\
174 & 23 & 0.70 & 0.30 & 816 & 810 & 805 \\
194 & 15 & 0.80 & 0.20 & 802 & 796 & 790 \\
192 & 13 & 0.82 & 0.18 & 799 & 793 & 788 \\
187 & 11 & 0.84 & 0.16 & 797 & 791 & 785 \\
199 & 10 & 0.86 & 0.14 & 794 & 788 & 782 \\
190 & 8 & 0.88 & 0.12 & 792 & 786 & 780 \\
204 & 7 & 0.90 & 0.10 & 790 & 782 & 778 \\
186 & 5 & 0.92 & 0.08 & 787 & 780 & 775 \\
203 & 4 & 0.94 & 0.06 & 784 & 779 & 772 \\
233 & 3 & 0.96 & 0.04 & 781 & 774 & 770 \\
159 & 1 & 0.98 & 0.02 & 779 & 772 & 768 \\
200 & 0 & 1.00 & 0.00 & 777 & 771 & 766
\end{tabular}




\section{$3 \quad$ References}

(1) Pelletier, M. J. Quantitative analysis using Raman spectrometry. Appl. Spectrosc. 2003, 57, 20A-42A, DOI: 10.1366/000370203321165133.

(2) Gmehling, J.; Kolbe, B.; Kleiber, M.; Rarey, J. R. Chemical thermodynamics for process simulation; Wiley-VCH-Verl.: Weinheim, 2012. 